journal club

\title{
Laserbehandlung der Onychomykose
}

\author{
Es wäre doch sehr schön und recht nützlich, über eine Alternative zur langwierigen lokalen und \\ oralen Behandlung der Nagelmykose mit ihren Problemen bei der Compliance und der Verträg- \\ lichkeit zu verfügen. Mit dem Laser gibt es eine mögliche Alternative.
}

\begin{abstract}
Originalia
1. Bhatta AK et al. Fractional carbon-dioxide (CO2) laser-assisted topical therapy for the treatment of onychomycosis. J Am Acad Dermatol 2016;74:916-23

2. Karsai $S$ et al Treating onychomycosis with the short-pulsed 1064-nm-Nd: YAG laser: results of a prospective randomized controlled trial JEADV 2017;31:175-80
\end{abstract}

Viele dermatologische und auch andere Praxen bieten ergänzend oder ausschließlich zur Behandlung des Nagelpilzes eine Lasertherapie an. Die bisherige Literatur bewertet die Ergebnisse jedoch teilweise recht widersprüchlich und eindeutige Resultate sind auch bisher nicht gesichert. Zwei neuere Arbeiten beschäftigen sich daher mit der Lasertherapie bei Onychomykose. In der Arbeit von Bhatta et al. aus Shanghai wurden insgesamt 75 Patienten mit 356 befallenen Nägeln untersucht [1]. Alle Nägel wurden mit dem $\mathrm{CO}_{2}$-Laser insgesamt dreimal im Abstand von vier Wochen gelasert und zusätzlich einmal täglich mit einer Terbinafin-Creme lokal für drei Monate therapiert.

Im Ergebnis waren nach drei Monaten knapp $95 \%$ der Patienten nativ (d.h. mikroskopisch) und $92 \%$ der Patienten kulturell negativ. Weitere drei Monate später sank diese Zahl jedoch wieder auf $84 \%$ beziehungsweise $80 \%$.
Wie die Autoren selbstkritisch bemerken, wies die Studie keine Kontrollgruppe auf und verfügte nur über eine recht kurze Beobachtungszeit von einem halben Jahr.

Demgegenüber betrug die Laufzeit der zweiten Studie ein ganzes Jahr und Karsai et al. setzten bei ihrer prospektiven kontrollierten Studie einen Nd:YAG-Laser ein [2]. Allerdings konnten in dieser monozentrischen Studie aus Deutschland nur 20 Patienten mit zusammen 82 befallenen Zehennägeln untersucht werden. Die Probanden wurden lokal am Fuß unter Aussparung der Nägel mit einem Antimykotikum (Loceryl ${ }^{\circledR}$ ) und dann entweder zusätzlich randomisiert mit dem Laser oder ohne zusätzliche Therapie behandelt.

Die Laserbehandlung selbst umfasste vier Termine im Abstand von 4-6 Wochen und als Endpunkt der Studie wurde eine komplette Remission der Onychomykose sowohl kulturell wie histologisch nach zwölf Monaten angestrebt. Im Ergebnis zeigte sich, dass keine der beiden Behandlungsabläufe ihr Ziel erreichte. Auch konnte keine optische Verbesserung (mit Hilfe eines spezifischen Onychomykose-Scores) dokumentiert werden. Die Autoren schlussfolgern daher, dass der Nd:YAG-Laser alleine langfristig keine positiven Effekte zeitigt. Seine Rolle als adjuvante Therapie sollte aber nach ihrer Einschätzung in weiteren Studien näher beleuchtet werden.

\section{- Kommentar}

\section{„Eine effiziente, rasche Therapie der Onychomykose scheint auf kurze Zeit nicht in Sicht zu sein"}

Im Jahre 2010 erteilte die US-amerikanische FDA dem gepulsten 1.064-nm-Nd:YAG-Laser die Zulassung bei der Behandlung der Onychomykose; allerdings primär unter dem Gesichtspunkt einer optischen beziehungsweise kosmetischen Verbesserung. In einer wissenschaftlichen Pilotstudie hatte die Anwendung des gepulsten Nd:YAG-Lasers zumindest eine Verbesserung des Onychomykose-Scores um $12 \%$ gezeigt, während In-vitro-Untersuchungen der biologischen Wirkung auf Trichophyton rubrum, dem häufigsten Erreger der Nagelmykosen, keine sicheren positiven Ergebnisse ergaben.

Von Teilen des Fachpublikums, vor allem aber von den Patienten wurde dieses Verfahren dennoch als rasch, unkompliziert, sicher und vor allem auch effizient eingeschätzt. Trotzdem gibt es weiterhin kontroverse Diskussionen über den Stellenwert und die Erfolgsaussichten dieser Methode. Während die zitierte chinesische Studie zumindest kurzfristig positive Resultate beim Lasern in Kombination mit einem topischen Antibiotikum (Terbinafin) nachweisen konnte, ergab die alleinige Behandlung mit dem Nd:YAG-Laser enttäuschen- de Therapieergebnisse trotz einer zusätzlichen Rezidivprophylaxe mit topischen Präparaten.

Für Kinderärzte, die ihre Patienten (und es gibt schon 2-jährige Kinder mit Nagelmykosen!) mit dieser Methode behandeln lassen wollen, lohnt sich also die Frage nach dem Design der Therapie und dem zusätzlichen Antimykotika-Einsatz. Und eine effiziente, rasche Therapie der Onychomykose scheint auf kurze Zeit leider auch nicht in Sicht zu sein.

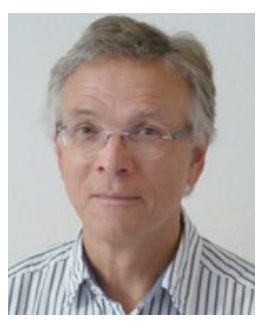

Dr. Ulrich Mutschler

Facharzt für Kinder- und Jugendmedizin 20146 Hamburg u.mutschler@gmx.de 